\title{
2014-2017 retrospective study on the analysis of causes of death, other than disease progression, in children diagnosed with acute lymphoblastic leukaemia - experience of the Paediatric Department of Fundeni Clinical Institute
}

\author{
Andreea Serbanica ${ }^{1,2}$, Letitia Radu1,2, Cristina Jercann ${ }^{1,2}$, Andra Beldiman², Ana Bica², \\ Petruta Vasilache ${ }^{2}$, Minodora Asan ${ }^{3}$, Oana Grigoras ${ }^{3}$, Anca Gheorghe ${ }^{3}$, \\ Codruta Popa ${ }^{1,3}$, Cerasela Jardan ${ }^{1,3}$, Constantin Arion ${ }^{1,2}$, Anca Colita1,2 \\ 1"Carol Davila" University of Medicine and Pharmacy, Bucharest, Romania \\ 2Paediatric Department, Fundeni Clinical Institute, Bucharest, Romania \\ ${ }^{3}$ Laboratory, Fundeni Clinical Institute, Bucharest, Romania
}

\begin{abstract}
Introduction. Acute lymphoblastic leukemia (ALL) is the most common form of cancer in the pediatric population (1). The survival rate has increased in recent years due to the continuous adjustment of therapeutic protocols (2). Despite all the efforts made alongside the evolution of new therapeutic protocols, the treatment related mortality (TRM) is around $2-4 \%$ in the Western European countries $(3,4)$.

Materials and methods. This retrospective is an analytical, observational and cohort-type study, the first of this kind in Romania, performed in a single pediatric hematology center, Clinical Institute Fundeni, between 2014 and 2017. It assesses the incidence of global mortality, the incidence of mortality due to any cause other than progression of disease (NRM $=$ Non Relapse Mortality) and the main cause of death in children diagnosed with ALL.

Results. We included 142 patients diagnosed between January 2014 and June 2017, with follow-up of 48 months post-diagnosis. The overall mortality of the cohort is $10.5 \%(15 / 142)$. The mortality rate for any other cause except disease progression (NRM) is $7.04 \%(10 / 15)$. These ten patients, in molecular remission, have as main cause of death complications that occurred during treatment (TRM= treatment related mortality with / without IRM = infection related mortality). The study showed increased percentages of IRM $6.3 \%$, over value of other studies, explaining and increasing also the value of NRM and also the value of global mortality; In contrast to TRM $2.1 \%$, this being in the reference range $(12,17,19,20)$. The most common cause of NRM was Clostridium difficile infection (4/10).

Conclusion. There are important to note the high percentage of achievement of complete remission (98\%) and impressive global survival at 4 years $(89.4 \%)$.
\end{abstract}

Keywords: acute lymphoblastic leukemia, mortality, survival, complications, children

\section{INTRODUCTION}

Acute lymphoblastic leukaemia (ALL) is the most common form of cancer in children, being a condition characterized by malignant proliferation of lymphoid cells blocked in primitive differentiation stages $(1,2)$. The current incidence of the dis- ease is increasing. In the United States the reported incidence is 41 to $1,000,000 / y e a r$, i.e. 3,100 new cases in children annually, and in Europe it varies according to the geographic area approximately 30/100,000/year in Eastern Europe and 40/100,000/ year in Western Europe $(3,4)$. 
In 2016, the American Cancer Society estimated an incidence of 6,590 new cases of ALL with a mortality of 1,400 cases/year (5).

Some of the multiple etio-pathogenic factors quoted as being associated with a high risk of developing ALL are: familial predisposition, associated genetic pathology, race, sex and external factors such as exposure to chemicals, ionizing radiation, pollution, infections, etc. these ones being under constant investigation $(6,7)$.

The significant increase of survival rates for patients with ALL from $10 \%$ in the 1970 s to over $80 \%$ at present, without the use of new drugs, has demonstrated the importance of including patients in randomized clinical trials that have allowed the permanent adaptation of therapeutic protocols to individual risk factors $(9,10)$.

Increasing the performance of supportive treatment correlate with the optimization of individual risk-adjusted chemotherapy protocols, the treatment-related mortality (TRM) rate is around $2-4 \%$ in the Western European countries $(11,12)$.

The multiple complications that may occur at the onset of the treatment are determined by both the pathology itself (complex blast proliferation with leukostasis) and complex chemotherapeutic treatment (secondary immunosuppression, infections, coagulation disorders, severe hydroelectrolytic and acido-basic, metabolic, neurological disorders etc.) influencing the survival by increasing morbidity and mortality (13).
This study, the first of this kind in Romania, conducted in a single pediatric hematology centre, Fundeni Clinical Institute, between January 2014 and June 2017, assesses the incidence of global mortality, the incidence of all-cause mortality other than dissease progression (NRM $=$ Non-Relapse Mortality) and the leading cause of death in children diagnosed with ALL.

\section{MATERIALS AND METHODS}

The study is an analytical, observational, retrospective, cohort-type study conducted at the Paediatric Haematology Department of Fundeni Clinical Institute for a period of 4 years. There were included patients whose cause of death was other than disease progression $(\mathrm{NRM}=$ Non-Relapse Mortality).

Patients diagnosed were classified in risk groups and monitored according to the ALL-BFM 2009 protocol, with the indication that L-asparaginase was administered instead of PEG-L-asparaginase, unavailable in Romania.

The diagnostic algorithm involves: anamnesis, clinical and para-clinical examination: peripheral blood smear count, identification of morphological, immune-phenotypic, cytogenetic and molecular characteristics of lymphoblasts on the onset, based on which subsequent monitoring is also performed - minimal residual disease (MRD). This

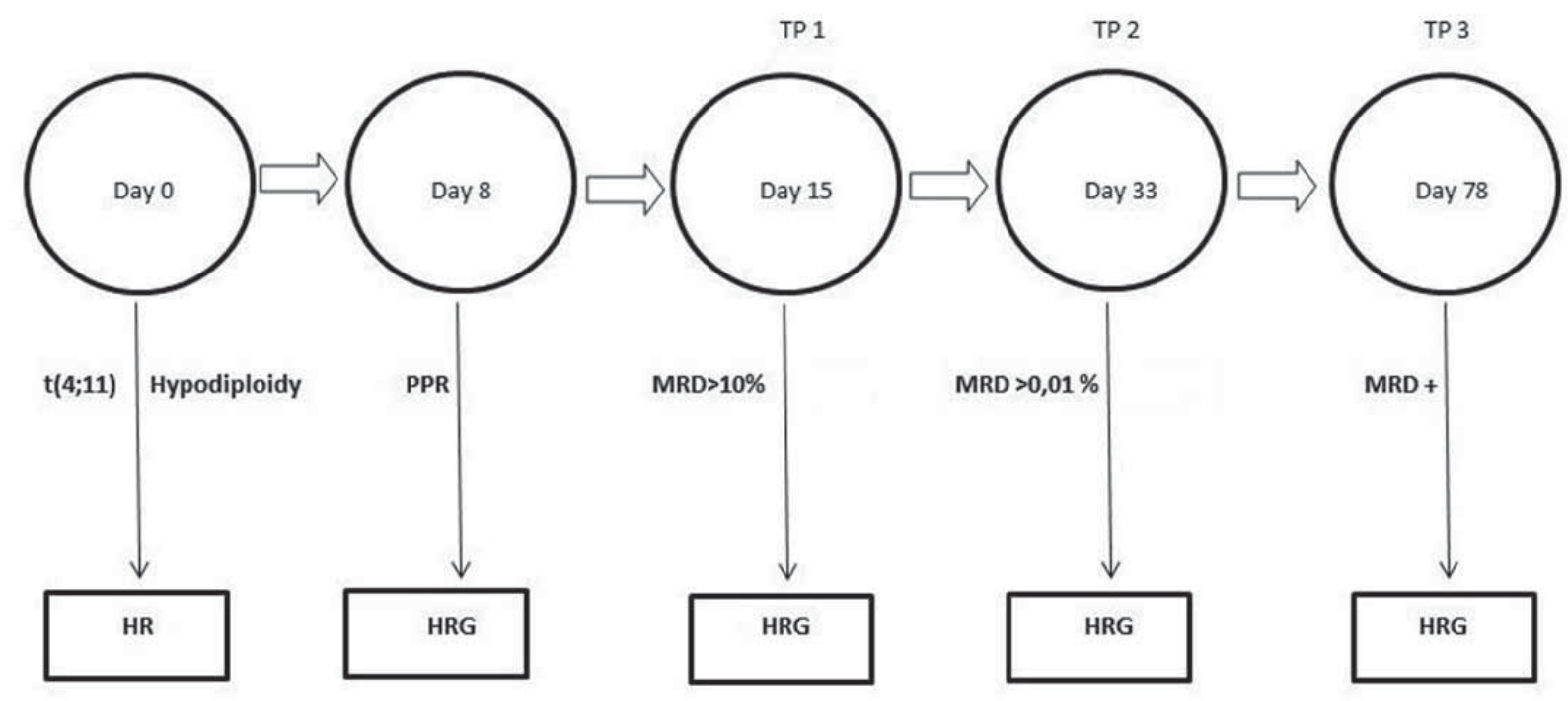


way we obtain a complete and complex diagnosis of the leukemic form, a stratification on risk groups, which leads to the establishment of an individualized treatment, drawing the premises of the prognosis in the long and short term.

Stratification on risk groups according to ALLBFM 2009 protocol includes the following parameters: age, leukocyte count at diagnosis, prednisone response, MRD on days 15, 33, 78 and leukemic cell genetics (structural and / or numerical abnormalities) $(14,15)$.

This classification on risk groups (see Fig. 1) requires personalized therapy per patient group with a decrease in mortality and morbidity rates. Adapting the degree of chemotherapy intensity (dose, frequency, duration) reduced toxicity without affecting the remission and the cure of acute leukaemia (13).

The chemotherapy treatment followed the 4 steps of the ALL-BFM 2009 protocol: induction, consolidation, reinduction and maintenance adapted to the appropriate risk degree.

Assessing the response to treatment on day 8 is an important parameter. According to studies, the level of blasts in peripheral blood on day 8 correlates with MRD for early classification in risk groups (see Fig. 1). MRD level on day 15 is directly proportional to the risk of relapse in children with ALL, the role of this parameter being a major one (see Fig. 2) (16).

Long-term monitoring of patients included in the study was performed by MRD, using flow cy- tometry method, FCM-MRD, and sought to identify relapses.

The measurable objectives were:

1) $\mathrm{NRM}=$ the total of deaths, of any other cause except from the disease progression, detected in ALL patients included in the study

$\mathrm{NRM}=\Sigma(\mathrm{TRM}+\mathrm{IRM})-$ Number of patients with progressive ALL / relapsed ALL.

2) $\mathrm{TRM}=$ treatment related mortality;

3) IRM = infection related mortality - para-clinically documented

The NRM was classified into two categories:

- the first one according to the time at which the death occurred, so we deduct a sub-classification a) NRM in induction = deaths occurring within the first 33 days after diagnosis and b) NRM after induction, after day 33 post-diagnosis

- a second one depending on the causes of death A) infection related mortality $=$ IRM and B) TRM = deaths from other complications except from infection and progression / recurrence of malignant disease.

We analysed the NRM for each risk group: standard, intermediate and high risk as well as the patient's therapeutic stages (induction and post-induction).

Calculation of cohort survival rate, progressionfree survival over the study period, and survival on risk groups are required in such a study given that the basic diagnosis is a malignant disease.

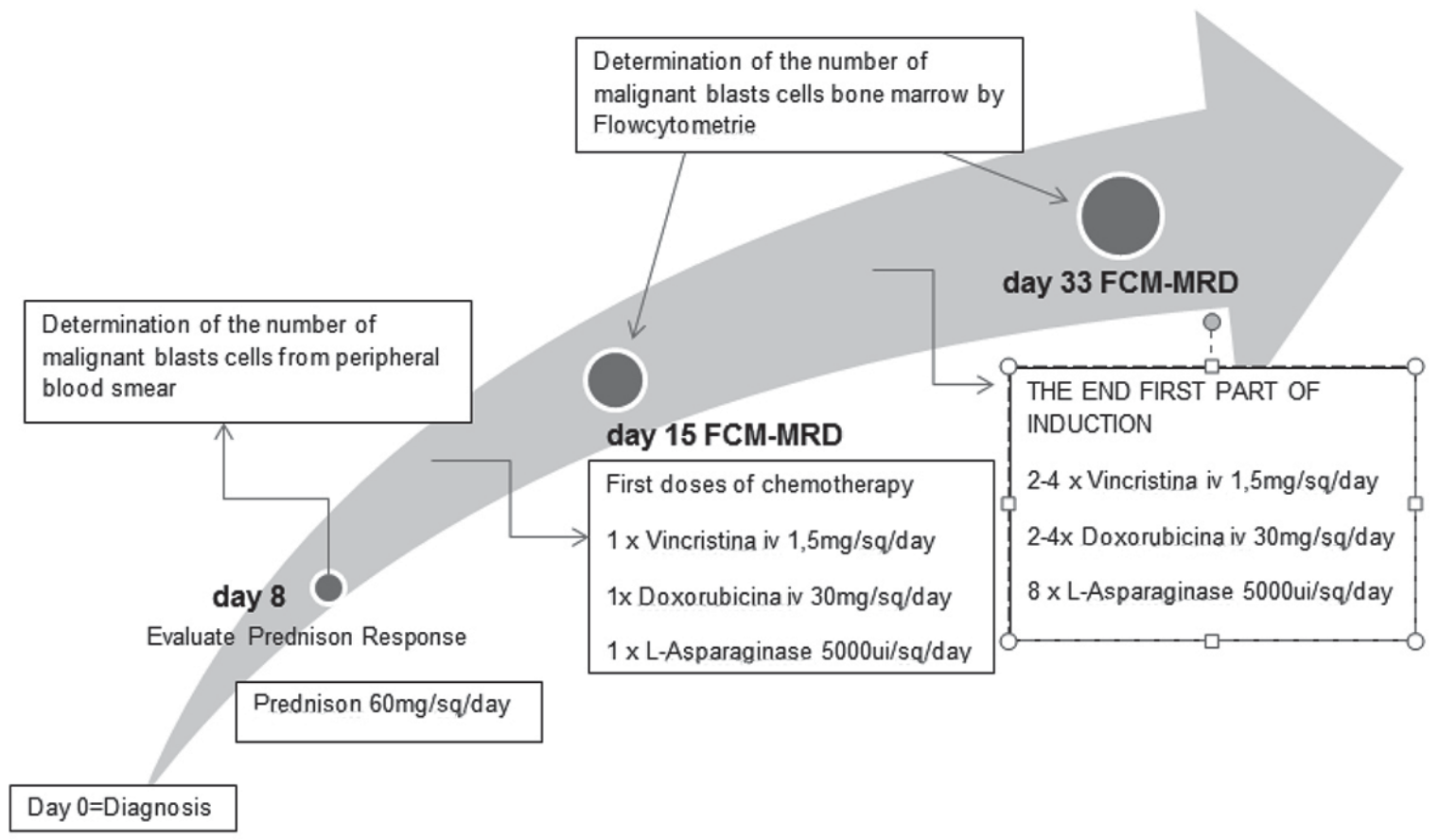

FIGURE 2. Patients' monitoring in the first part of the induction - adaptation according to the ALL-BFM protocol 
Patient data were extracted from the medical records and with the help of Hipocrate's operator, which were later included in the Word-Excel 2010 program.

The calculation and graphic lay-out of the survival curves used the Kaplan-Meier method, and the comparison of the TRM incidence on the risk groups was performed with the Chi square test.

\section{RESULTS}

The study was conducted in a single haematological centre, the Paediatrics Department of Fundeni Clinical Institute; it included a total of 142 patients diagnosed with ALL between January 2014 and June 2017, monitored until 31.12.2017. Of these, $10(7.04 \%)$ patients died of other cause than disease progression (NRM), and 5 patients had progressive malignancies and were excluded from this study. The characteristics of the cohort are shown in Table 1.

TABLE 1. Statistical data on the number, epidemiological characteristics, diagnosis, initial response to treatment and classification on risk groups

\begin{tabular}{|l|c|c|}
\hline $\begin{array}{l}\text { General characteristics of the } \\
\text { cohort }\end{array}$ & Values & $\%$ \\
\hline $\begin{array}{l}\text { Total number of pa ents diagnosed } \\
\text { with ALL between January 2014 - } \\
\text { June 2017 }\end{array}$ & 142 & $100 \%$ \\
\hline Environment of origin Urban / Rural & $86 / 56$ & $60.5 \% / 39.5 \%$ \\
\hline Sex Male / Female & $80 / 62$ & $56.3 \%$ / 43.6\% \\
\hline Age average (range 1-17) & 6.5 & - \\
\hline B cell / T cell ALL & $119 / 23$ & $83.8 \% / 16.2 \%$ \\
\hline $\begin{array}{l}\text { PGR / PPR (Prednisone Good / Poor } \\
\text { Responder) }\end{array}$ & $110 / 32$ & $77.5 \% / 22.5 \%$ \\
\hline $\begin{array}{l}\text { Risk groups SRG = standard risk } \\
\text { group }\end{array}$ & 57 & $40.14 \%$ \\
\cline { 2 - 3 } $\begin{array}{l}\text { BFM 2009IRG = intermediate risk } \\
\text { group } \\
\text { HRG = high risk group }\end{array}$ & 43 & $30.28 \%$ \\
\cline { 2 - 3 } & & $29.57 \%$ \\
\hline
\end{tabular}

The distribution of the included patients supports the increased incidence of ALL in urban areas, in male sex, and the increased frequency of $\mathrm{B}$ cell proliferation as compared with $\mathrm{T}$ cell proliferation, and it is consistent with published literature $(12,14)$.

All deceased patients were diagnosed with Bcell ALL (10/10), the form with a more favourable prognosis, and a lower mortality rate than T-cell ALL (see Table 2).

TABLE 2. Final percentage results related to the mortality in the cohort

\begin{tabular}{|l|c|}
\hline FINAL RESULTS & VALUES \\
\hline \% Incidence of mortality in this cohort & $10.5 \%$ \\
\hline \% Another cause of mortality except from & $7.04 \%$ \\
progression of the disease (NRM) & \\
\hline \% Mortality on risk groups SRG & $3.5 \%$ \\
\cline { 2 - 2 } IRG & $9.3 \%$ \\
\hline HRG & $9.5 \%$ \\
\hline \% Overall survival at 4 years & $89.4 \%$ \\
\hline
\end{tabular}

The overall mortality of the cohort includes all causes of mortality involving the diagnosis of acute lymphoblastic leukaemia. The value obtained is a $10.5 \%(15 / 142)$ figure which is comparatively higher with BFM data (see Table 2) (15).

The mortality rate of any other cause except for disease progression (NRM) is 7.04\% (10/15). These patients have the treatment-related mortality as main cause of death (TRM = treatment related mortality with / without IRM = infection related mortality) (see Table 3).

Determination of the mortality on risk groups and of the treatment stage in which most of the patients died revealed a $4.2 \%$ of induction mortality rate $(6 / 142)$, the period known with the highest mortality risk among all treatment stages (see Table 3) (17). Death of patients during induction had a value above that of recent studies (17).

The mortality of those in complete remission, post-induction NRM (> day 33) was 2.8\% (4/142) being in the range quoted by other studies (16).

The study demonstrates an increased mortality rate of $9.5 \%$ (4/42) also in the high risk ALL group. NRM calculation performed on risk groups demonstrated values with no statistical significance $(\mathrm{p}>0.05)$.

TABLE 3. Mortality on risk groups / treatment stages

\begin{tabular}{|c|c|c|c|c|c|c|}
\hline $\begin{array}{c}\text { Risk } \\
\text { groups }\end{array}$ & $\begin{array}{c}\text { No. of } \\
\text { pa ents/ } \\
\text { risk groups }\end{array}$ & $\begin{array}{c}\text { Deaths } \\
\text { in induc on }\end{array}$ & $\begin{array}{c}\text { Deaths in } \\
\text { consolida on/ } \\
\text { re-induc on }\end{array}$ & $\begin{array}{c}\text { Infec on related } \\
\text { mortality } \\
\text { (IRM) }\end{array}$ & $\begin{array}{c}\text { NRM mortality/ } \\
\text { risk groups }\end{array}$ & $\begin{array}{c}\text { Mortality on risk } \\
\text { groups }\end{array}$ \\
\hline SRG & 57 & 1 & 1 & 2 & 2 & $\mathbf{3 . 5 \%}$ \\
\hline IRG & 43 & 3 & 1 & 2 & 4 & $\mathbf{9 . 3 \%}$ \\
\hline HRG & 42 & 2 & 2 & 4 & 4 & $\mathbf{9 . 5 \%}$ \\
\hline- & $\mathbf{1 4 2}$ & $\mathbf{6}$ & $\mathbf{4}$ & $\mathbf{8}$ & $\mathbf{1 0}$ & $\mathbf{7 . 0 4 \%}$ \\
\hline
\end{tabular}




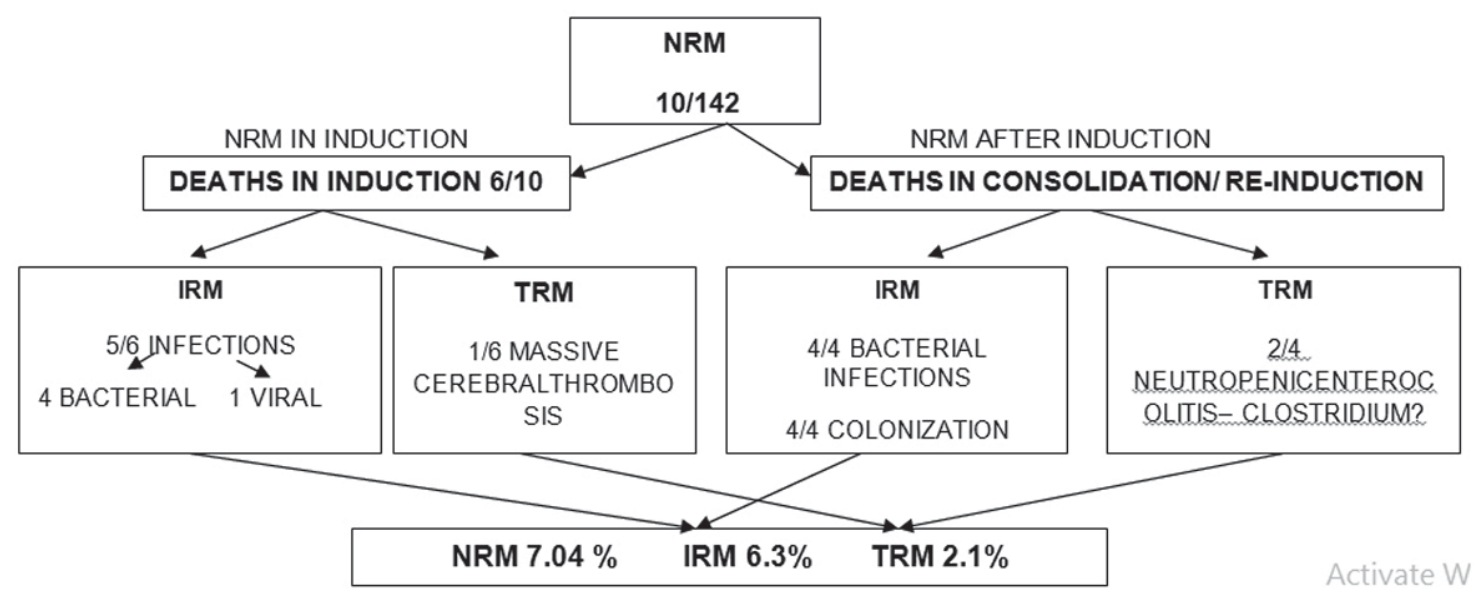

FIGURE 3. Final results by type of mortality

Event-free survival (EFS) is represented in Diagram 1. EFS in the standard group is $93.8 \%$ and HRG around $83 \%$, which is a value comparable to that reported in the BFM protocol.

The overall survival rate of the cohort at 4 years is even higher compared to EFS. (see Fig. 5).

\section{DISCUSSIONS}

Determining the exact cause of death for each patient is difficult to identify due to the multitude of simultaneous post-chemotherapy complications per patient that precipitates the infection, thus demonstrating the close cause-and-effect relationship between TRM and IRM.

This aspect was also revealed by Hadel Hassan et al. (19), which presents a new mortality classification system to demonstrate the common denominator between these notions: NRM, IRM, TRM; Because The toxicity of chemotherapy produces immunosuppression that precipitates the infection, so IRM is secondary to TRM and together they pro-

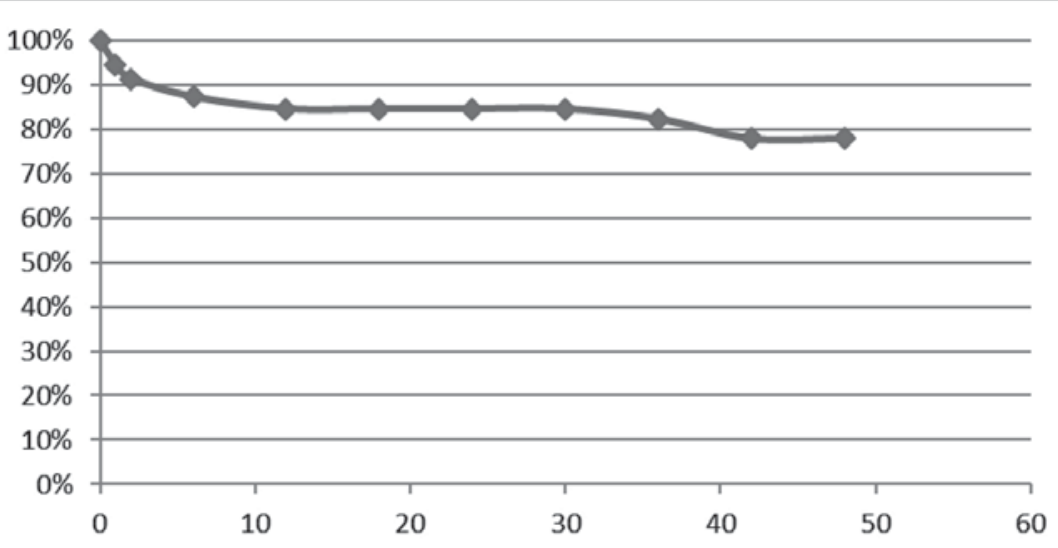

FIGURE 4. Event-free survival of the cohort at 4 years

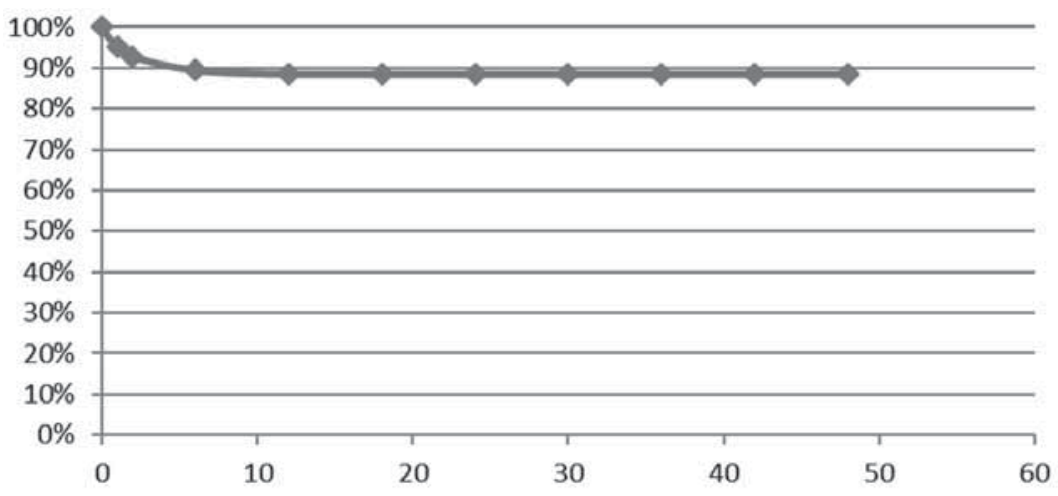

FIGURE 5. Global cohort survival at 48 months (4 years) from diagnosis 
duce NRM, the notion of TRM becomes a general term valid for any cause of death not directly related to cancer, excluding progression or recurrence of the disease (19). In this study, we used the notions in the above-defined sense, in the material and methods section, precisely to identify the most common cause of mortality other than progression of disease / relapse.

$\mathrm{NRM}=$ total deaths, of any other cause, except for disease progression, detected in patients with AL included in the study; So NRM includes TRM + IRM

$\mathrm{TRM}=$ mortality due to treatment-related complications, excluding infection, progression / recurrence of malignancy

IRM $=$ infection related mortality - para-clinically documented

The most common acute complications with a direct impact on mortality in these patients (TRM) were: post-chemotherapy aplasia (10/10), neutropenic enterocolitis $(4 / 10)$ with/without toxic megacolon and/or intestinal perforation, (3/10), neurological disorders (thrombosis / ischemia / cerebral haemorrhage 3/10, PRESS, SIADH 4/10), metabolic disorders (hyperglycaemia +/- diabetes insipidus $1 / 10$ ) and severe hydro-electrolytic disorders (hyponatremia, hypokalaemia 10/10). All ten patients had simultaneusly multiple post-chemotherapy complications.

Chemotherapy by adverse reactions and by cumulative toxicity raises the occurrence of infection, which, despite antibiotic, prophylactic and curative treatment, leads to sepsis (IRM). The starting point of infections in a neutropenic child severely immunocompromised in the context of chemotherapy is hard to identify. For this reason, antibiotic, antifungal and antiparasitic prophylaxis, as well as regular screening of the bacterial port, is part of the therapeutic protocol of such a patient. Within the cohort, the most common outbreaks of infections were found in the digestive tract $(5 / 10)$, followed by those in the ENT sphere (transmission in nasal and / or pharyngeal exudates 3/10). Dysmicrobisms occurring in conjunction with the strictly hygienicdietetic oncology regimen, intensive chemotherapy associated with antibiotic therapy, long-term hospitalization, and high immunosuppression have led to digestive colonisations with the following bacteria: Enterococcus sp. + / VRE +, ESBL + E. coli, Clostridium and nasal or pharyngeal transmission with Staphylococcus MRSA, Pseudomonas aeruginosa, Acinetobacter, Stenotrophomonas maltophila (see Fig. 6). These are possible starting points for sepsis and influence the mortality rate by infections. At the same time, it has been observed that Enterococci $s p+/ \mathrm{VRE}+$ binding precipitates Clostridium difficile infection.

The most common cause of death was Clostridium difficile infection (4/10) despite continued prophylactic administration of metronidazole therapy and, when necessary, of vancomycin orally / intravenously (see Fig. 2).

Despite the efforts made in terms of hygiene, patient isolation, prophylactic and curative treatments, most of the deaths were due to infections. $8 / 10$ bacterial infections, $1 / 10 \mathrm{flu}$, and $1 / 10$ patients died of massive cerebral thrombosis non-responsive to pharmacological and surgical therapy, associating with evolution of sepsis with Acinetobacter. In conclusion, we can say that all ten patients presented concomitant infections, thus confirming the difficulty of establishing the exact cause of mortality and the close cause-and-effect relationship of the two major categories of TRM and IRM (see Fig. 7).

The most common cause of NRM was nosocomial infection.

The increased IRM percentage of $6.3 \%$ compared to the outcomes of the study conducted by David O'Connor et al. draws the attention, although

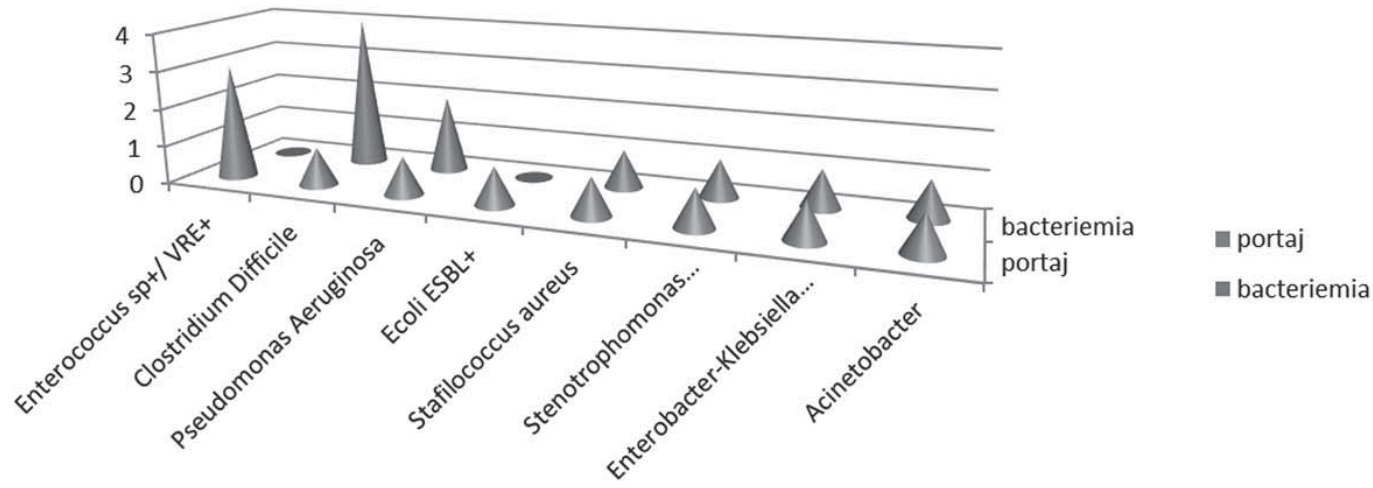

FIGURE 6. Graphic representation of transmissions / bacteriemia in deceased patients 


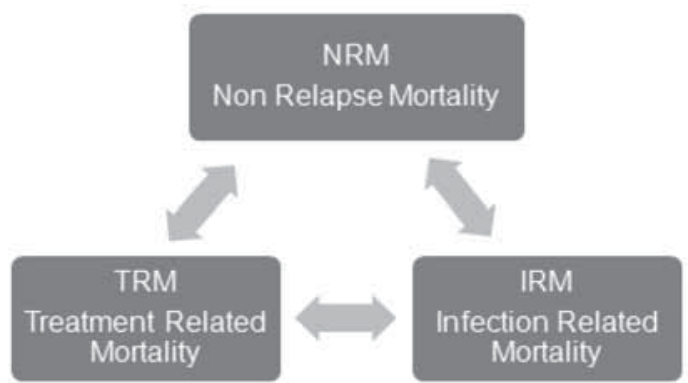

FIGURE 7. The close cause-and-effect relationship of the two major categories of TRM and IRM

the $2.1 \%$ TRM value is one in the reporting range $(20,21)$.

The increased IRM values of the study, both in induction (5/10/142) and after this therapeutic period $(4 / 10 / 142)$, led to the conclusion that infections (IRM) are the main source of the mortality percentage increase, of any other cause except from disease progression (NRM).

The value represented on our NRM group is $7.04 \%$, increased compared to the data presented by the study conducted by Blanco E et al. (17), reporting an incidence of $3.6 \%$. The source of difference is the increased percentage, of approximately two-three times, of infection-related mortality (IRM), as evidenced by our study compared to others.

\section{REFERENCES}

1. Pui CH, Evans WE. Treatment of Acute Lymphoblastic Leukemia. NEJM 354160, ian 2006

2. SEER Cancer Statistics Review, National Cancer Institute. Bethesda, MD. http://seer.cancer.gov/faststats/selections.php?\#Output (Accessed on feb 2018).

3. Howlader N, Noone AM, Krapcho M. SEER Cancer Statistics Review (CSR) 1975-2013. Bethesda, Md: National Cancer Institute, 2015.

4. Special section: Cancer in children and adolescents. In: American Cancer Society: Cancer Facts and Figures 2014. Atlanta, Ga: American Cancer Society, 2014, pp 25-42 https://www.cancer.org/ content/dam/cancer-org/research/cancer-facts-and-statistics/ annual-cancer-facts-and-figures/2014/special-section-cancer-inchildren-and-adolescents-cancer-facts-and-figures-2014.pdf

5. Spector LG, Ross JA, Robison LL et al. Epidemiology and etiology. In: Pui $\mathrm{CH}$, editor. Childhood Leukemias. New York: Cambridge University Press;2006.p.48-66].

6. Terwilliger T, Abdul-Hay M et al. Acute Lymphoblastic Leukemia: A comprehensive review and 2017 update, Blood Cancer, jun2017; 7(6);e577; https://www.ncbi.nlm.nih.gov/pubmed/28665419]

7. Joshua Yew Suang Lim, Smita Bhatia et al. Genomics of Racial and Ethnic Disparities in Childhood Acute Lymphoblastic Leukemia. Cancer. 2014 Apr 1; 120(7): 955-962.; https://www.ncbi.nlm.nih.gov/ pmc/articles/PMC4015138/

8. Wartenberg D, Groves FD, Adelman AS. Acute lymphoblastic leukemia: Epidemiology and etiology. In: Estey EH, Faderl S, Kantarjian H, eds. Acute Leukemias. 1st ed. Berlin, Germany: Springer; 2008:77-93.

9. Gupta $S$ et al. Treatment related mortality in children with acute lymphoblastic leukemia in Central America, Cancer 2011
On the chosen group there were no differences between NRMs on risk groups during the induction or remission period, unlike the data presented in the meta-analysis conducted by Blanco E et al. showing a lower NRM during the remission period for patients at standard risk.

\section{CONCLUSIONS}

Currently, infections are the leading cause of death in children with acute lymphoblastic leukaemia in remission, despite all prophylactic measures. The most common cause of death was nosocomial infection with Clostridium difficile (4/10).

The infection related mortality rate is increased (IRM 6.3\%), thus the mortality rate of any other cause has increased, except from the progression of the disease (NRM 7.04\%) and the overall mortality rate of the cohort $(10.5 \%)$.

However, it is important to note the high percentage of achieving complete remission (98\%), as well as the survival at 4 years of $89.4 \%$. Survival is high compared to reported data including by the BFM consortium with a relative difference in the patients with ALL of the high-risk group HRG.

\section{Conflict of interest: none declared Financial support: none declared}

10. Smith MA, Altekruse SF, Adamson PC et al. Declining childhood and adolescent cancer mortality. Cancer. 2014 Aug 15;120(16):2497-506 doi: 10.1002/cncr.28748. Epub 2014 May 22- last accesoct 2018 https://www.ncbi.nlm.nih.gov/pubmed/24853691?dopt=Abstract

11. Gatta G, Capocaccia R, Stiller $C$ et al. Childhood cancer survival trends in Europe: A EUROCARE Working Group study. J ClinOncol $2005 ; 23: 3742$

12. Terzah M Horton, $C$ Philip Steuber et al. Overview of the treatment of acute lymphoblastic leukemia in children and adolescents. www. uptodate.com, uptodated in July 2017, accessed January 2018 (https://www.uptodate.com/contents/overview-of-the-presentationand-diagnosis-of-acute-lymphoblastic-leukemia-in-children-andadolescents.

13. Colita Anca, Colita Andrei. Leucemia acuta limfoblastica la copii. Bucuresti, Editura Universitara "Carol Davila", 2014.

14. Terzah M Horton, $C$ Philip Steuber et al. Risk group stratification and prognosis for acute lymphoblastic leukemia în children and adolescents. www.uptodate.com, uptodated in Jun 2017, accessed January 2018

15. AIEOP-BFM ALL 2009 studio, Versione 1.1, 15 Aprile 2010 Protocollo di diagnosi e terapia della leucemia linfoblastica acuta în eta' pediatrica. Sponsor: University Hospital Schleswig-Holstein, Campus Kiel, Brunswiker Str. 10, 24105 Kiel, Germany

16. Borowitz MJ et al. Clinical significance of minimal residual disease in chidhood acute lymphoblastic leukemia and its relationship to other prognostic factors: A Children's Oncology Group study. Blood. 111, 5477-5485 (2008). 
17. Blanco $E$ et al. Non-relapse mortality in pediatric acute lymphoblastic leukemia: A systematic review and meta-analysis. Leuk Lymphoma, volume 53, mai 2012

18. Maaz Aur, Badar F, Mehmood T et al. High infection related mortality in Pakistani children with ALL during remission induction chemotherapy: Review of data from single institution. Journal of Cancer\&Alliedspecialities, dec 2016, www.journals.sfu.ca/jcas/article/ view/94/html]].

19. Hadeel Hassan, Rompola M et al. Validation of classification system for treatment-related mortality in children with cancer, BMJ Pediatrics Open 2017].
20. David O'Connor, Jessica Bate, Rachel Wade et al. Infection-related mortality în children with acute lymphoblastic leukemia: An analysis of infectious deaths on UKALL2003. blood journal 14.08.2014, 124: 1056-1061 (http://www.bloodjournal.org/content/ bloodjournal/124/7/1056.full.pdf?sso-checked=true)

21. Hanafy Ahmed H, Rawaa Solaiman, Dalia Bilal et al. Early Deaths in pediatric acute leukemia; a challenge in developing countries, Blood 2016] 